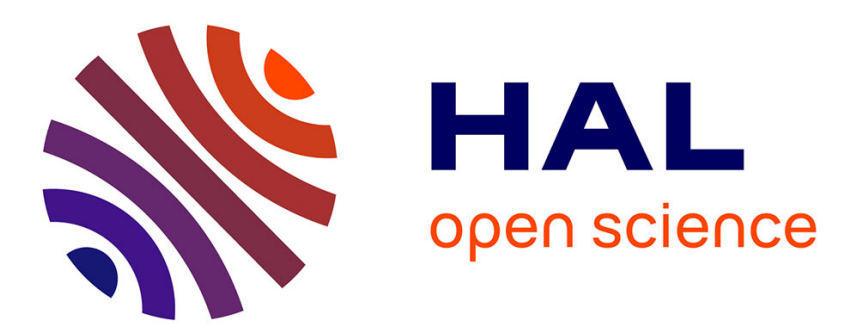

\title{
Reconstitution des crues extrêmes du Gardon à partir d'une analyse paléohydrologique
}

\author{
L. Dezileau, B. Terrier, J.F. Berger, P. Blanchemanche, A. Latapie, R.
} Freydier, André Paquier, M. Lang, J.L. Delgado

\section{- To cite this version:}

L. Dezileau, B. Terrier, J.F. Berger, P. Blanchemanche, A. Latapie, et al.. Reconstitution des crues extrêmes du Gardon à partir d'une analyse paléohydrologique. La Houille Blanche - Revue internationale de l'eau, 2014, 4, pp.44-52. 10.1051/lhb/2014037 . hal-01108400

\section{HAL Id: hal-01108400 \\ https://hal.science/hal-01108400}

Submitted on 18 Feb 2015

HAL is a multi-disciplinary open access archive for the deposit and dissemination of scientific research documents, whether they are published or not. The documents may come from teaching and research institutions in France or abroad, or from public or private research centers.
L'archive ouverte pluridisciplinaire HAL, est destinée au dépôt et à la diffusion de documents scientifiques de niveau recherche, publiés ou non, émanant des établissements d'enseignement et de recherche français ou étrangers, des laboratoires publics ou privés. 


\title{
Reconstitution des crues extrêmes du Gardon à partir d'une analyse paléohydrologique
}

\author{
DEZILEAU L.. ${ }^{*}$, TERRIER B. ${ }^{2}$, BERGER J. F. ${ }^{3}$, BLANCHEMANCHE P. ${ }^{4}$, LATAPIE A. ${ }^{5}$, FREYDIER R. ${ }^{6}$, \\ PAQUIER A. ${ }^{5}$, LANG M. ${ }^{5}$, DELGADO J.L. ${ }^{7}$ \\ 1. Geosciences Montpellier, Université Montpellier 2, CNRS, UMR 5243, France. \\ 2. Agence de l'eau Rhône-Méditerranée et Corse, Lyon cedex, France. \\ 3. Environnement Ville et Société, Université Lumière Lyon 2, IRG/UMR 5600, CNRS, France. \\ 4. Archéologie des Sociétés Méditerranéennes, CNRS, UMR 5140, France \\ 5. Irstea, Unité de recherche hydrologie Hydraulique, CS70077, Villeurbanne Cedex, France. \\ 6. Hydrosciences Montpellier, Université Montpellier 2, CNRS, UMR 5569, France. \\ 7. CETE Méditéranée, Aix-en-Provence, France \\ * Corresponding author: Present address: UMR 5243 CC60 UM2/CNRS, Place E. Bataillon 34095 Montpellier cedex 5, France. \\ Fax: +33 (0) 4671449 30,E-mail adresse: laurent.dezileau@gm.univ-montp2.fr
}

\begin{abstract}
RÉSUMÉ. - Une étude paléohydrologique a été entreprise dans les Gorges du Gardon, où deux coupes sédimentaires ont été étudiées. La première provient d'une terrasse (coupe GE située à $10 \mathrm{~m}$ de hauteur au dessus de la rivière) et l'autre, d'une cavité (coupe GG localisée à $15 \mathrm{~m}$ au-dessus de la rivière). L'identification et la mise en évidence des différents paléo-événements de crue ont été établies grâce à l'inspection minutieuse de chaque dépôt sédimentaire et d'une étude géochronologique fine $\left({ }^{137} \mathrm{Cs},{ }^{210} \mathrm{~Pb}_{\mathrm{ex}},{ }^{14} \mathrm{C}\right.$, métaux traces, objets archéologiques, historiques des crues). Nous avons souhaité évaluer la capacité des terrasses fluviatiles du Gardon à enregistrer des paléo-évènements en comparant les coupes sédimentaires aux données historiques des hauteurs d'eau et aux résultats d'un modèle hydraulique 1D. Nous montrons que la terrasse GE enregistre la quasi-totalité des évènements du $20^{\text {ème }}$ siècle et qu'à priori, ce type d'objet géomorphologique peut être utilisé pour toute étude paléohydrologique sur le Gardon. De plus, l'étude de la coupe GG (cavité perchée à $15 \mathrm{~m}$ ) permet de reconstituer les évènements intenses au cours des 600 dernières années. Nous montrons une augmentation des crues extrêmes dans le Haut-Languedoc au cours du « Petit âge glaciaire ».
\end{abstract}

Mots-clés : Paleohydrologie, crues extrêmes, Gardon, Petit Age Glaciaire

\section{Paleohydrological reconstruction of extreme floods of the Gardon River, SE France}

\begin{abstract}
This paper presents a paleohydrological study conducted on sediment flood deposits at two sites located in the Gardon Gorges, Southeast France. The first study site is a valley-side terrace (referred to as site GE, located $10 \mathrm{~m}$ above the channel bed) and the second is located in a cave (referred to as site GG, perched $15 \mathrm{~m}$ above the bed level). Paleo-flood events were identified by carefully analyzing each slackwater flood deposits. The flood units were then dated using a variety of dating techniques, namely ${ }^{137} \mathrm{Cs},{ }^{210} \mathrm{~Pb}_{\mathrm{ex}},{ }^{14} \mathrm{C}$ geochemical analysis of mining-contaminated slackwater flood sediments, archeological objects and the historic flood record. Hydraulic modeling was also carried out in order to verify the coherence between the stratigraphic records of flood deposits at the sites and the historic flood levels known along the river Gardon. It is demonstrated that all the major flood events of the $20^{\text {th }}$ century can be identified at terrace GE. This type of geomorphological unit can therefore be used as a sound basis for a paleohydrological analysis of the Gardon Gorges. Furthermore, the analysis of the stratigraphic record at site GG demonstrates that the site can be used to trace the largest flood events that have occurred within the past 600 years. The study reveals that the frequency of extreme flood events increased during the Little Ice Age.
\end{abstract}

Key-words: Paleohydrology, extreme floods, Gardon, Little Ice Age

\section{INTRODUCTION}

Les événements de crue extrêmes sont, par nature, rares. L'hydrologue a peu de chance de disposer localement d'une grande collection d'observations, alors que le principe de " l'analyse probabiliste des valeurs extrêmes » suppose d'étudier une (longue) série d'événements. On en déduit un " comportement probabiliste » que l'on extrapole ensuite à l'ensemble de la population des crues ou des pluies fortes. On se trouve dans la pratique confronté à deux difficultés :
- Comment extrapoler des séries trop courtes, ne disposant le plus souvent d'informations que sur des évènements plus ou moins courants (absence d'événement " extrême ») ? - Comment mettre à jour notre connaissance des évènements extrêmes en intégrant les évolutions éventuelles liées au changement du climat?

La paléohydrologie peut apporter des réponses à ces questions. Pour cela, les dépôts laissés par les crues, soit directement dans le champ d'inondation, soit dans des grottes qui surplombent le lit mineur sont étudiés (Benito 
and Thordycraft, 2005). Ces études permettent de reconstituer le niveau atteint par les plus fortes crues depuis plusieurs millénaires. Sur le bassin de l'Ardèche, Sheffer et al., (2003) ont montré que la crue de 1890, la plus forte connue sur une période historique de quatre siècles, était également la crue la plus importante enregistrée depuis plusieurs milliers d'années. Ces approches permettent donc d'élargir de façon considérable l'échelle chronologique d'investigation et donnent des éléments objectifs sur les crues majeures survenues sur le bassin versant. Notre travail va se concentrer principalement sur le Gardon, zone d'étude pour laquelle il existe une quantité importante de données historiques, particulièrement sur les pluies et débits des 100 dernières années. Nous utilisons des techniques d'analyse stratigraphique et de datation des dépôts $\left({ }^{137} \mathrm{Cs},{ }^{210} \mathrm{~Pb},{ }^{14} \mathrm{C}\right.$, géochimie des polluants, archéologie) pour évaluer l'altitude atteinte par les eaux lors des plus forts événements, et proposer une chronologie des crues du Gardon. Des modélisations hydrauliques unidimensionnelles sont également utilisées pour calculer la valeur de débit minimale permettant d'atteindre les coupes étudiées.

\section{SITE D'ÉTUDE ET HYDROLOGIE}

Le bassin versant des Gardons (1858 $\mathrm{km}^{2}$ à Remoulins) est situé au sud est du Massif Central. Le Gardon fait 135 $\mathrm{km}$ de long depuis sa source au Mont Lozère (1699 m d'altitude) jusqu'à sa confluence avec le Rhône à $6 \mathrm{~m}$ d'altitude (Figure 1a.). Au niveau géologique, les Cévennes sont caractérisées par des formations géologiques de l'ère primaire et sont principalement composées de granite, de schiste, de gneiss et de grès (Bonnifait et al., 2009). En aval, le Gardon traverse la plaine du Gard qui est caractérisée par des roches carbonatées du secondaire (Figure 1b). Dans cette zone, les formations massives du Crétacé ont forcé la rivière à créer de profonds canyons (les Gorges du Gardon). Ces formations calcaires présentent un haut degré de karstification. Plus en aval encore, les formations secondaires sont recouvertes par les sédiments fluviatiles Quaternaire du Rhône.

Le Gardon a un régime typiquement méditerranéen, avec un faible débit annuel moyen $\left(33 \mathrm{~m}^{3} / \mathrm{s}\right.$, SAGE des Gardons, 2000), d'importantes variations saisonnières et des pointes de crue de plus de 100 fois son débit moyen. Les précipitations annuelles moyennes sont de 900 à $1100 \mathrm{~mm}$ sur la partie supérieure du bassin et de 500 à $700 \mathrm{~mm}$ sur la partie inférieure. Nuissier et al., (2008) fournissent une analyse détaillée des caractéristiques de crues éclair dans cette région. Des précipitations importantes peuvent s'accumuler sur le bassin du Gardon pendant plusieurs jours, en particulier à la fin de l'été et au début de l'automne. Ces perturbations d'origines frontales sont bloquées et renforcées par la présence des hauts reliefs du Massif Central. Lorsqu'un système méso-échelle convectif reste stationnaire pendant plusieurs heures, de fortes pluies de plus de $200 \mathrm{~mm}$ peuvent être enregistrées en moins d'une journée et donc conduire à des inondations dévastatrices dans cette région. De nombreuses données hydrologiques sont disponibles auprès du service de prévision des crues (SPC30) et de l'autorité locale (« Smage des Gardons »). La station de mesure située à Remoulins (environ $15 \mathrm{~km}$ en aval des sites d'étude) fournit des mesures de hauteur d'eau depuis 1890 (figure 2). Depuis le début des mesures, trois grandes inondations ont été enregistrées avec des hauteurs d'eau supérieures à $7 \mathrm{~m}$ et des débits estimés à plus de $5000 \mathrm{~m}^{3} / \mathrm{s}$ : le 16 et 17 Octobre $1907\left(5300 \mathrm{~m}^{3} / \mathrm{s}\right)$, le 4 Octobre 1958 $\left(6400 \mathrm{~m}^{3} / \mathrm{s}\right)$ et les 8 et 9 Septembre $2002\left(7000 \mathrm{~m}^{3} / \mathrm{s}\right)$. Cette dernière crue extrême a provoqué la mort de 23 personnes et causé 1,2 milliards d'euros de dommages aux villes et villages le long du fleuve. Sept mille maisons ont été endommagées dont 100 complètement détruites, 1500 ont été submergées sous $2 \mathrm{~m}$ d'eau (Huet et al., 2003).
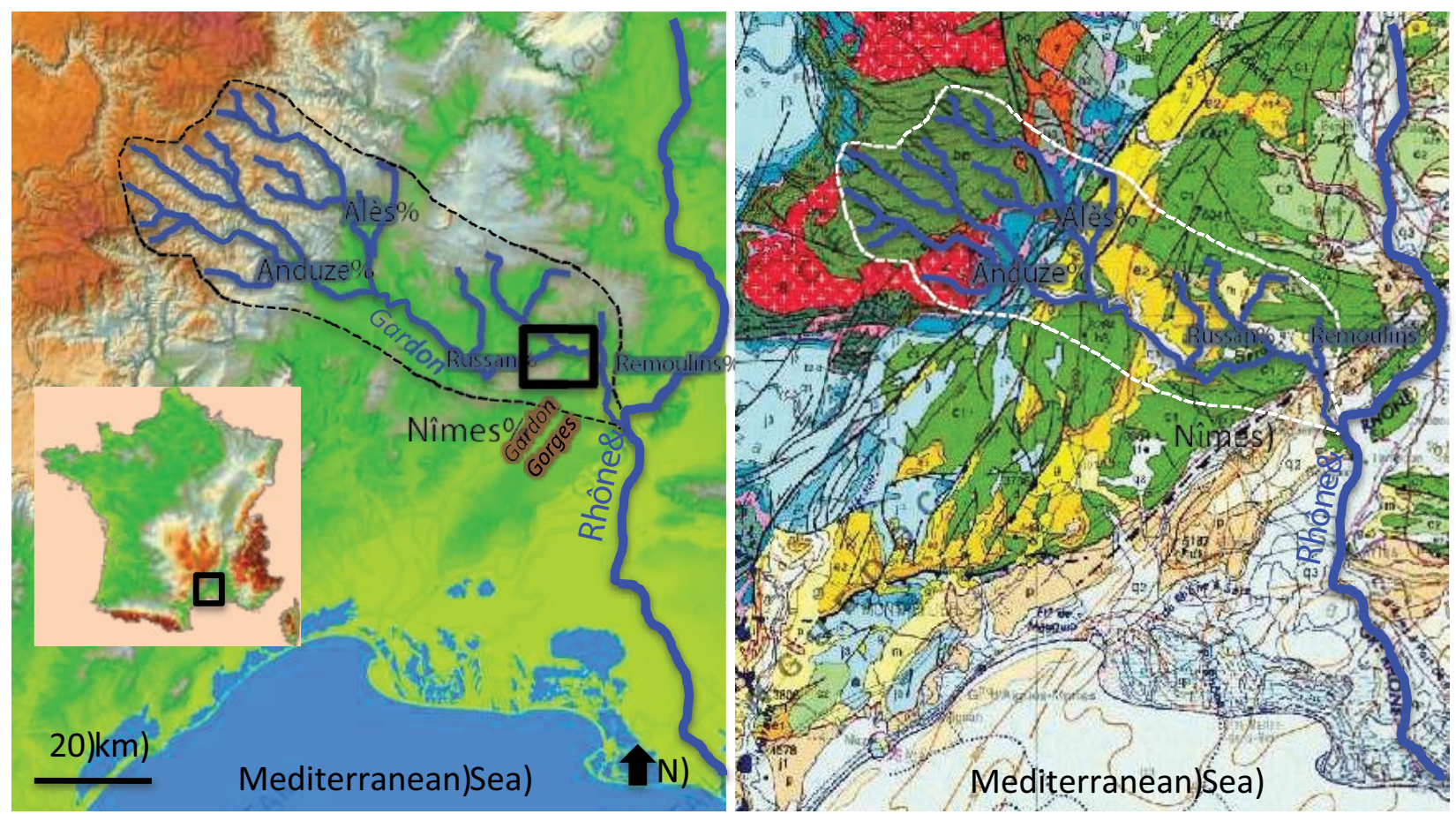

Figure 1a et b : Topographie, hydrographie et carte géologique du Gardon. 


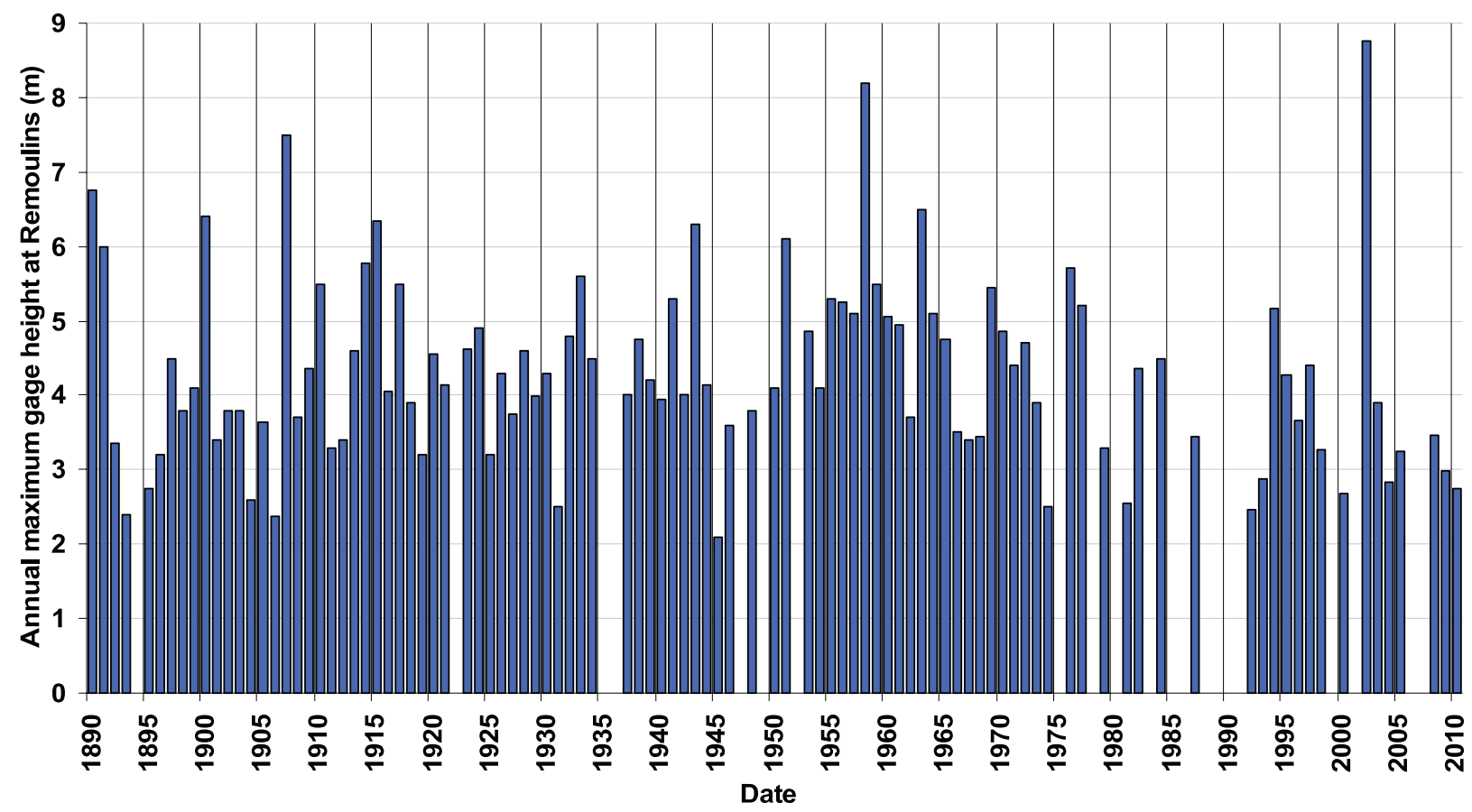

Figure 2 : Hauteurs maximales annuelles disponibles à Remoulins entre 1890 et 2010.

\section{MÉTHODES}

\section{III.1. Mise en évidence des dépôts de crue du passé}

Deux coupes ont été étudiées le long du Gardon (Figure 3), elles se localisent dans la partie centrale des Gorges entre Collias et le Pont Saint Nicolas. Ces coupes proviennent d'une terrasse (GE) et d'une cavité (GG). La première coupe GE a été choisie pour tester et valider l'approche paléohydrologique en croisant les résultats obtenus avec les informations de la station hydrométrique de Remoulins située juste en aval des gorges. Le niveau de cette coupe (GE : altitude $+10 \mathrm{~m}$ au-dessus du lit de la rivière) correspond à des crues courantes. La seconde coupe GG avait pour but de recenser des crues plus fortes, et est donc située à une altitude plus élevée (cavité à $+15 \mathrm{~m}$ au-dessus du lit de la rivière). L'identification et la mise en évidence des différents paléo-événements de crue a été établie grâce à l'inspection minutieuse de chaque dépôt sédimentaire (décompte des lamines, identification de paléosols, détection de surfaces d'érosion, indication de bioturbation, présence de niveaux de cailloutis dans la cavité ou couches d'occupation anthropique entre différents événements d'inondation, étude de la variabilité granulométrique à partir d'un granulomètre laser Beckman Coulter LS 13320).

\section{III.2. Géochronologie}

Toute reconstitution paléo-environnementale, à partir de l'étude des archives sédimentaires, nécessite l'établissement d'une échelle chronologique la plus fine possible, afin de dater avec une bonne résolution, les événements de crue du passé. Les isotopes de prédilections pour dater les évènements du dernier siècle sont $l e{ }^{210} \mathrm{~Pb}$ et le ${ }^{137} \mathrm{Cs}$. Ils permettent une datation précise, grâce à leurs propriétés chimiques (réactivité vis-à-vis des particules) et physiques (périodes de désintégrations, rayonnement gamma). Le ${ }^{137} \mathrm{Cs}$ et le ${ }^{210} \mathrm{~Pb}$ sont mesurés par spectrométrie gamma (détecteur CANBERRA BEGe 3825) à Géosciences Montpellier.

\section{III.2.1. Datation par le ${ }^{210} \mathrm{~Pb} b_{e x}\left(T_{1 / 2}=22,3\right.$ ans $)$}

Le Plomb 210 est un isotope radioactif naturel issu de la chaîne de désintégration de l'uranium 238. L'uranium 238, contenu naturellement dans la croûte terrestre, subit la série de désintégration suivante :

Uranium $238 \rightarrow$ Uranium $234 \rightarrow$ Thorium $230 \rightarrow$ Radium $226 \rightarrow$ Radon 222

Le ${ }^{222} \mathrm{Rn}\left(\mathrm{T}_{1 / 2}=3,8\right.$ jours) ainsi produit, se trouve sous une forme gazeuse. Lorsqu'il est produit à proximité de la surface, le gaz s'échappe dans l'atmosphère où il se transforme rapidement en Polonium 218 qui se désintègre en quelques minutes en ${ }^{210} \mathrm{~Pb}$. Ce ${ }^{210} \mathrm{~Pb}$, dit atmosphérique (ou en excès) retombe sous forme de particules humides ou sèches et s'accumule dans les sédiments. Son activité au sein de la colonne sédimentaire diminue avec le temps selon une loi de décroissance radioactive. Le principe de la datation au ${ }^{210} \mathrm{~Pb}$ s'appuie sur l'hypothèse selon laquelle le flux atmosphérique en ${ }^{210} \mathrm{~Pb}$ est constant, ce qui implique que l'activité de l'isotope radioactif à la surface des sédiments reste toujours identique. Cette hypothèse permet en mesurant l'activité $\mathrm{du}{ }^{210} \mathrm{~Pb}$ et en appliquant la loi de décroissance radioactive de déterminer l'âge de nos sédiments. Au bout de cinq fois sa période, c'est à dire 110 ans, il a complètement disparu dans l'environnement. La mesure $\mathrm{du}{ }^{210} \mathrm{~Pb}$ sur le détecteur gamma CAMBERRA se fait en intégrant le pic à $46,5 \mathrm{keV}$. $\mathrm{Le}^{210} \mathrm{~Pb}$ ex est estimé en soustrayant le pic du ${ }^{210} \mathrm{~Pb}$ au pic du ${ }^{226} \mathrm{Ra}$ à $186,2 \mathrm{keV}$. L'erreur n'excède pas $6 \%$.

\section{III.2.2. Datation par le ${ }^{137} \mathrm{Cs}\left(T_{1 / 2}=30,17\right.$ ans $)$}

Le Césium 137 est un isotope radioactif artificiel issu de réactions de fission nucléaires. Il est relâché dans l'atmosphère lors de l'utilisation de bombes atomiques et d'accidents dans des centrales nucléaires. Avec une période de demi-vie (30,17 ans) proche de celle $\mathrm{du}{ }^{210} \mathrm{~Pb}$, il permet de dater les séquences sédimentaires sur une même échelle temporelle. Les profils d'activité du Césium 137 en fonction de la profondeur dans une coupe sédimentaire présentent des pics dont le plus important 

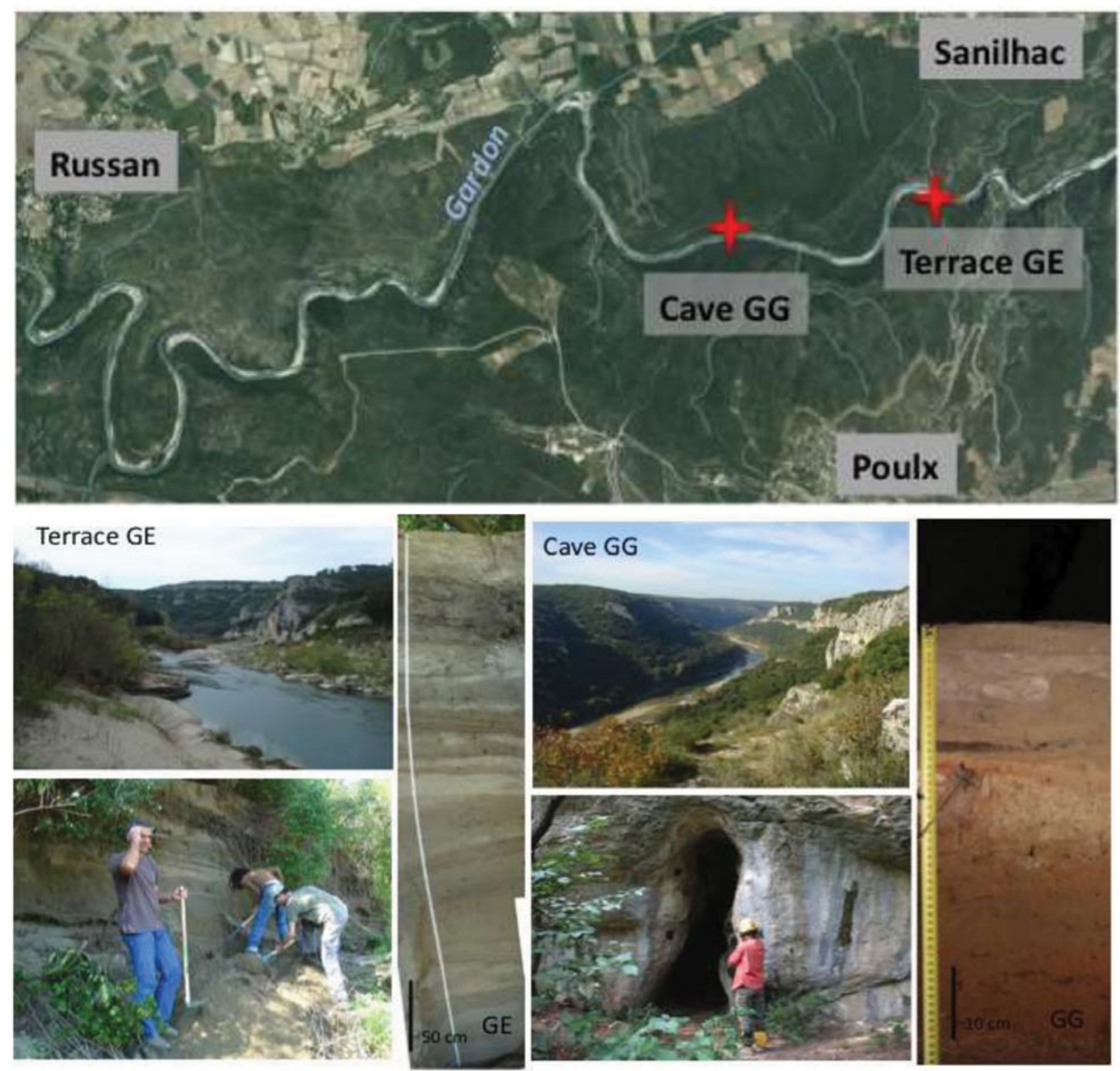

Figure 3: (a) Carte des sites d'étude; (b) Terrasse GE et Cavité GG avec les coupes des archives sédimentaires correspondantes.

est généralement attribué à l'année 1963, date du maximum de rejets atmosphériques par les essais nucléaires. La présence de Césium 137 dans les dépôts sédimentaires indiquera qu'ils ont été déposés postérieurement aux années 1950. La mesure $\mathrm{du}{ }^{137} \mathrm{Cs}$ sur le détecteur gamma CAMBERRA se fait en intégrant le pic à $661 \mathrm{keV}$. L'erreur n'excède pas $6 \%$.

\section{III.2.3. Datation grâce à l'historique des pollutions en plomb du Gardon}

L'historique des pollutions en plomb sur le bassin versant du Gardon étant connu, les concentrations en plomb ont été mesurées afin d'affiner la chronologie des dépôts sur les périodes les plus récentes. Les concentrations en $\mathrm{Al}$ et en $\mathrm{Pb}$ ont été déterminées après digestion totale des sédiments à l'aide d'un ICP-MS, XSeries II (Thermo Fisher Scientific), équipé d'une CCT (Collision Cell Technology) au Laboratoire HydroSciences Montpellier. Pour savoir s'il y a eu un enrichissement du $\mathrm{Pb}$, un facteur d'enrichissement (FE) a été calculé. Les niveaux de base du plomb et de l'aluminium correspondent aux concentrations du $\mathrm{Pb}$ et de l'Al dans les niveaux de crue les plus anciens. Le calcul du FE de plomb inclut sa normalisation par rapport à l'aluminium suivant l'équation: $\mathrm{FE}_{\mathrm{Pb}}=(\mathrm{Pb} / \mathrm{Al})_{\text {échantillon }} /(\mathrm{Pb} / \mathrm{Al})_{\text {base naturelle }}$.

\section{III.2.4. Datation par le ${ }^{14} \mathrm{C}\left(T_{1 / 2}=5600\right.$ ans $)$}

Afin de compléter la chronologie sur des périodes de temps plus long des échantillons de charbons de bois ont été sélectionnés et des analyses ${ }^{14} \mathrm{C}$ ont été réalisées au $\mathrm{LMC} 14$ (Laboratoire de Mesure ${ }^{14} \mathrm{C}$ sur ARTEMIS à l'institut du CEA à Saclay (Commissariat de l'Energie Atomique). Les mesures de ${ }^{14} \mathrm{C}$ ont été converties en âges calendaires (Tableau 1) en utilisant le programme CALIB 6.1.0 (Stuiver and Reimer 1993).

\section{III.3. Le modèle hydraulique unidimensionnel}

Un modèle hydraulique unidimensionnel (Rubar3, El Kadi Abderrezzak et Paquier 2009) a été construit sur le secteur des Gorges afin d'associer un débit aux cotes des dépôts identifiés par l'approche paléo-hydrologique. La géométrie 
Tableau 1 : Ages $14 C$ BP et âges calendaires $(A D)$ de la coupe $G G$.

\begin{tabular}{|l|c|c|c|}
\hline \multicolumn{1}{|c|}{ Nom } & Profondeur (cm) & Age 14C BP & Age Calendaire (AD) \\
\hline GG30-32 & 31 & $355+/-30$ & $1574-1626$ \\
\hline GG39 & 39 & $430+/-30$ & $1421-1499 ?$ \\
\hline GG63 & 63 & $415+/-30$ & $1440-1482$ \\
\hline GG68-70 & 69 & $415+/-30$ & $1440-1482$ \\
\hline GG80 & 80 & $490+/-30$ & $1418-1439$ \\
\hline GG80-85 & 82 & $425+/-30$ & $1437-1472$ \\
\hline Bar A 87-90 & 88 & $395+/-30$ & $1445-1494$ \\
\hline Bar A 110-115 & 113 & $580+/-30$ & $1317-1353$ \\
\hline
\end{tabular}

du modèle est basée sur les données topographiques disponibles après 1999, fournies par le SPC30 et le Smage des Gardons ainsi que des levés spécifiques réalisés dans le cadre du projet ANR Extraflo (2009-2013). Les coefficients de rugosité du modèle ont été calés sur la crue de 2002 et validés sur la crue de $1958(\Delta \mathrm{H} / \mathrm{H}<10 \%)$.

\section{RESULTATS ET DISCUSSION}

\section{IV.1. Estimation du débit de submersion des sites par le modèle hydraulique 1D}

L'objectif de cette comparaison est d'associer un débit aux cotes des dépôts identifiés par l'approche paléohydrologique. Dans un premier temps une succession de régimes permanents est injectée en entrée du modèle unidimensionnel afin d'extraire des simulations les relations hauteurs-débits au droit des sites. Des tests de sensibilité avec des variations de débits et des variations du coefficient de rugosité de $\pm 10 \%$ sont effectués afin de fournir une enveloppe et donc une gamme de débits associée à chaque dépôt.

La relation hauteur-débit calculée avec le modèle unidimensionnel pour la cavité GG est présentée sur la Figure 4 . Les variations de débit de $\pm 10 \%$ influent peu sur les résultats de hauteurs d'eau, en revanche les variations du coefficient de rugosité permettent d'obtenir une enveloppe de la courbe de tarage au droit du site. L'eau affleure le seuil du site pour un débit compris entre $4050 \mathrm{~m}^{3} / \mathrm{s}$ et $4825 \mathrm{~m}^{3} / \mathrm{s}$.
La relation hauteur-débit calculée avec le modèle unidimensionnel pour la coupe GE montre que l'eau affleure le haut de la coupe pour un débit compris entre $1700 \mathrm{~m}^{3} / \mathrm{s}$ et $2100 \mathrm{~m}^{3} / \mathrm{s}$.

Les gammes de débits de submersion calculées pour la cavité GG et la terrasse GE sont reportées sur la chronique de débits reconstituée à la station hydrométrique de Remoulins. L'historique des hauteurs d'eau en crue disponibles a été converti en débit avec la courbe de tarage fournie par le SPC 30 (courbe no. V1-11). Il faut souligner que cette courbe de tarage correspond aux estimations hautes de la crue de 2002 où le pic de crue est généralement estimé entre $6500 \mathrm{~m}^{3} / \mathrm{s}$ et $7200 \mathrm{~m}^{3} / \mathrm{s}$ (Sogreah, 2003). En se basant sur cette reconstitution de la chronique de débit, le nombre d'événements ayant atteint les sites depuis 1890 peut-être déterminé (Figure 5). Trois événements semblent ainsi avoir distinctement submergé la cavité GG (1907, 1958 et 2002). Pour la terrasse GE, 21 événements sont identifiés comme ayant submergé la terrasse. Ces résultats nécessitent d'être mis en parallèle avec les paléo-évènements de crue.

\section{IV.2. Stratigraphie sédimentaire et chronologie}

\section{IV.2.1. La terrasse GE}

La terrasse GE est constituée de 20 unités individuelles de dépôts de crue (notées de GE1 à la base à GE20 au sommet, figure 3 et 6 ). La figure 6 présente les unités de dépôts de crue identifiées, la granularité, les activités de ${ }^{210} \mathrm{~Pb}_{\mathrm{ex}}$, de
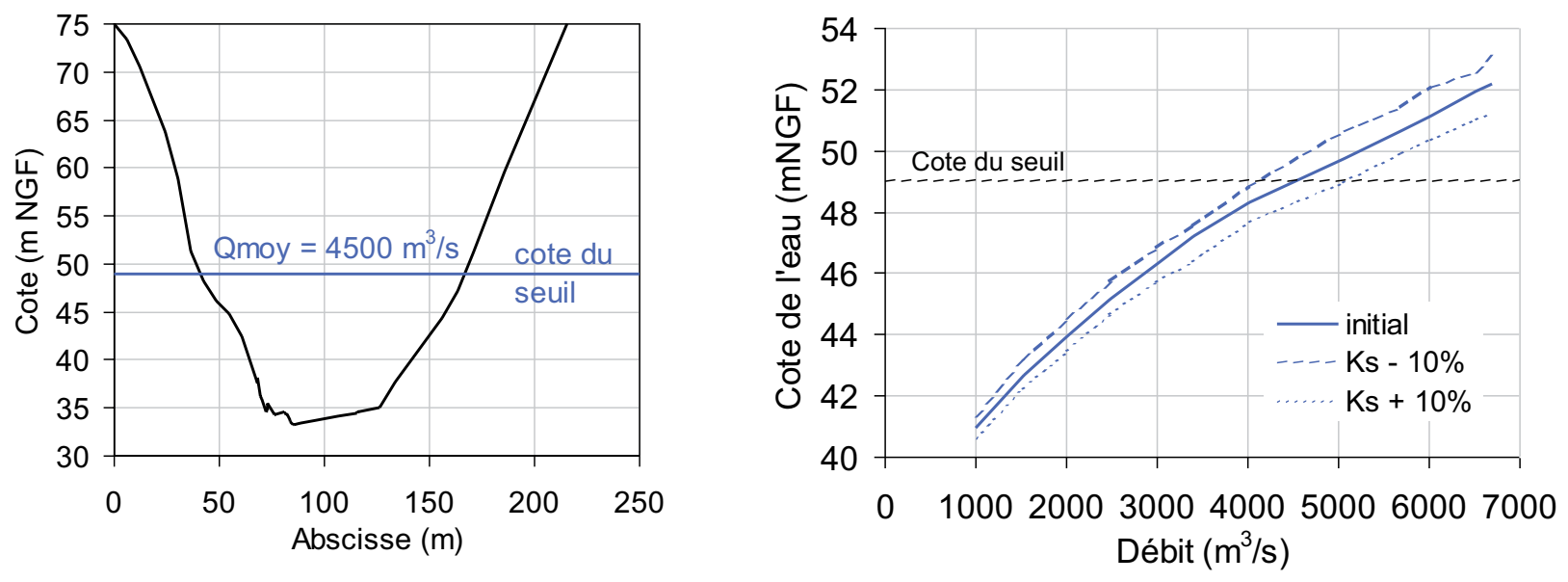

Figure 4 : Cavité $G G$ : (a) profil en travers au droit de la cavité et (b) relation hauteur-débit calculée au droit du site. 


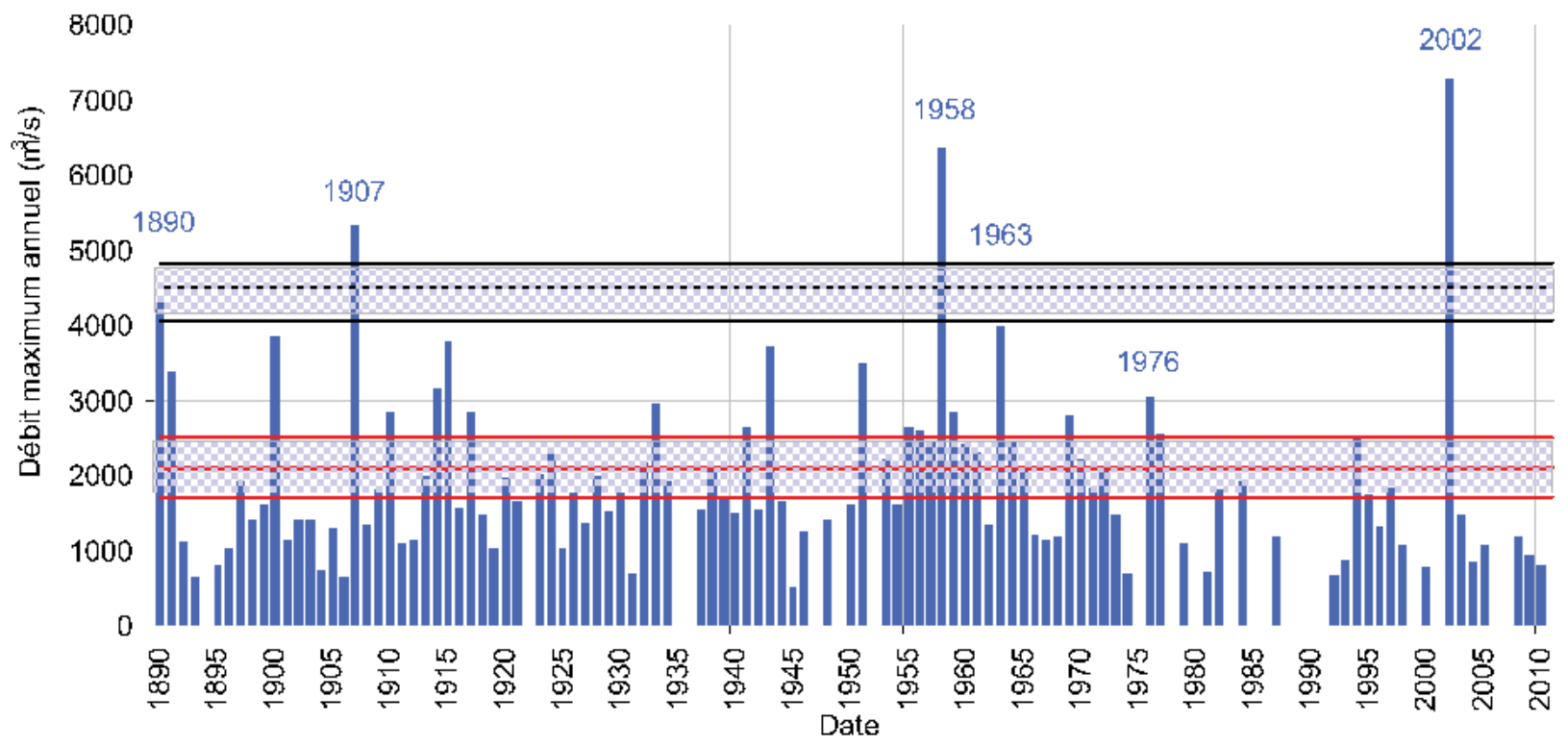

Figure 5 : Historique des débits de crue reconstitués à Remoulins et enveloppe des débits de submersion de la cavité GG (en noir) et de la terrasse GE (en rouge).

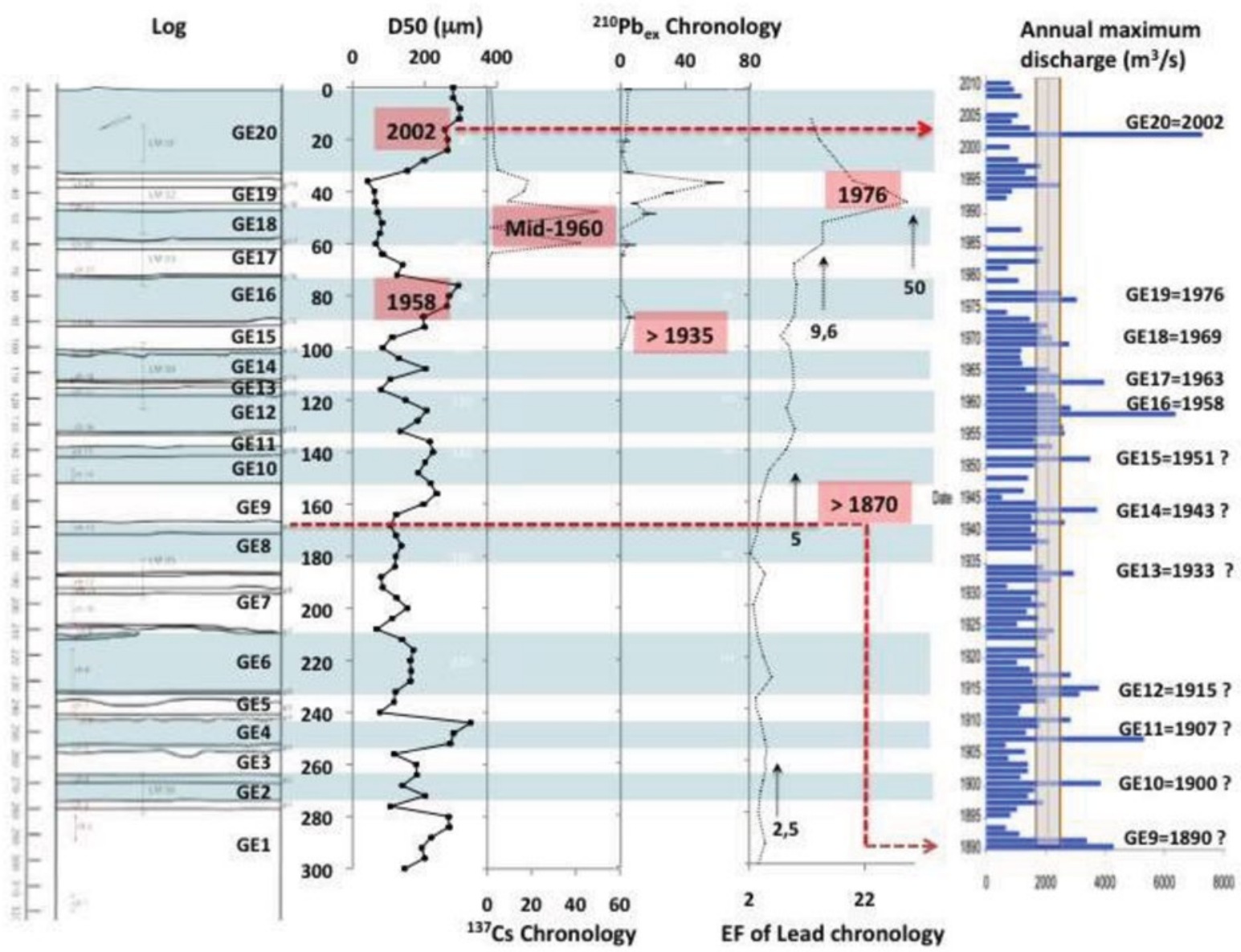

Figure 6 : Log lithostratigraphique des dépôts de crue. Chaque dépôt de crue est annoté par deux lettres (GE) et un chiffre qui suit l'ordre stratigraphique. Modèle d'âge pour les dépôts de crue de la terrasse GE : Les fortes granulométries (D50) des dépôts GE20 et GE16 nous permettent d'identifier les évènements de 2002 et 1958 ; Les pics d'activités du ${ }^{137} \mathrm{Cs}$ entre 40 et $60 \mathrm{~cm}$, nous permettent d'associer les évènements de crue GE17 et GE18 aux années 60 ; La présence de ${ }^{210} \mathrm{~Pb} b_{e x}$ à partir de $90 \mathrm{~cm}$ suggère que les évènements GE15 à GE20 sont postérieurs aux années 35 ; Le facteur d'enrichissement du plomb nous permet d'identifier la crue de 1976 caractérisée par une forte pollution du gardon (FE=50) mais aussi de mettre en évidence les évènements postérieurs aux années 1870 (FE > 5) correspondant au début de l'exploitation minière dans la région; L'historique des débits mesurés à Remoulins permet d'affiner l'identification chronologique des évènements sédimentaires de crue. L'enveloppe du débit minimum pouvant submerger le site est présentée en grisé. 
${ }^{137} \mathrm{Cs}$, le facteur d'enrichissement en $\mathrm{Pb}$ et l'enveloppe $\mathrm{du}$ débit minimum pouvant submerger le site GE.

Le césium-137 est présent dans les unités GE17, GE18, GE19 et GE20, avec des valeurs maximales de 38 et $45 \mathrm{mBq} / \mathrm{g}$ dans les unités GE17 et GE18, respectivement. Le Césium 137 est un isotope radioactif artificiel produit principalement par les essais nucléaires atmosphériques et il est présent dans l'environnement à partir des années 55 . Dans la séquence des dépôts de crue de la terrasse GE, les sédiments déposés après 1955 peuvent être identifiés grâce $\mathrm{au}^{137} \mathrm{Cs}$ (Figure 6). Le premier événement postérieur à 1955, identifié par les premières traces de ${ }^{137} \mathrm{Cs}$, est représenté par l'unité GE17, indiquant que les 4 autres dépôts de crue GE17-GE20 sont tous postérieurs à cette date. D'autre part, les fortes activités en césium-137 mesurées dans les unités GE17 et GE18, (38 et $45 \mathrm{mBq} / \mathrm{g}$ ) peuvent être associées à la production maximale de césium-137 atmosphérique au milieu des années 1960 (autour de 1963, figure 6).

La première unité contenant $\mathrm{du}{ }^{210} \mathrm{~Pb}_{\mathrm{ex}}$ est l'unité GE15 située à $90 \mathrm{~cm}$ de profondeur dans le profil stratigraphique, avec une valeur de $5 \mathrm{mBq} / \mathrm{g}$. Le ${ }^{210} \mathrm{~Pb}_{\mathrm{ex}}$ est présent dans les unités GE15, GE17, GE18, GE19 et GE20, avec une valeur maximale de $58 \mathrm{MBq} / \mathrm{g}$ dans l'unité GE19. La demi-vie du plomb-210 est de 22,26 ans et pourrait théoriquement permettre de dater par décroissance radioactive des dépôts sédimentaires sur les 110 dernières années. Cependant, dans ce type d'environnement et compte tenu des barres d'erreur sur les mesures, nous estimons que la méthode du plomb-210 ne peut pas être utilisée pour dater des dépôts de crue supérieurs à 80 ans. La méthode $\mathrm{du}^{210} \mathrm{~Pb}_{\text {ex }}$ peut nous permettre de confirmer un certain nombre de résultats obtenus à l'aide $\mathrm{du}^{137} \mathrm{Cs}$. L'activité la plus élevée de ${ }^{210} \mathrm{~Pb}_{\mathrm{ex}}$ enregistrée dans l'unité GE19 et la décroissance exponentielle de ce radioélément dans les autres dépôts de crue sous-jacents (GE18 à G15) confirment que la stratigraphie n'est pas perturbée. De plus, la première trace de ${ }^{210} \mathrm{~Pb}_{\mathrm{ex}}$ dans le profil est mesurée dans l'unité GE15, ce qui indique que les 6 dépôts de crue GE15-GE20 sont récents et probablement tous postérieurs aux années 1930 (Figure 6).

Le facteur d'enrichissement (FE) du Pb présente une variation très forte entre la base et le sommet de la terrasse, avec des valeurs comprises entre 2,5 et 50 (Figure 6). Les valeurs les plus basses (autour de 2,5) caractérisent les unités de GE1 à GE9. Le FE est plus élevé dans les unités supérieures de la terrasse avec des valeurs de 5 entre GE10 et GE17, de 9 dans l'unité GE18 et de 50 dans l'unité GE19. Le FE diminue dans la dernière unité GE20 (8). L'analyse géochimique de sédiments contaminés par l'exploitation minière, dans des plaines d'inondation, a été largement utilisée pour dater des dépôts de crue lorsque l'historique de l'activité minière est connue (Knox et Daniels, 2002; Thorndycraft et al, 2004). L'extraction du plomb et du zinc sur le haut bassin versant du Gardon a commencé en 1730 (Elbaz-Poulichet et al., 2006). Le nombre de concessions minières a considérablement augmenté entre 1860 et 1930 . Pendant cette période, l'activité minière a généré 400000 tonnes de déchets. Entre 1951 et 1963, les sociétés minières Pennaroya puis Metaleurop ont largement exploité le minerai produisant entre 2300000 et 5000000 de tonnes de déchets (30 000 tonnes de plomb et 3500 tonnes de Zinc). Cette activité minière s'est arrêtée en 1993. Une des mines les plus importantes de la région est la mine de Carnoules qui a généré au total plus de 1500000 tonnes de déchets. La mine a officiellement fermée le 24 Octobre 1963. En Septembre 1976, le barrage d'un terril s'est brusquement ouvert suite à un épisode cévenol et $100000 \mathrm{~m}^{3}$ d'eau contenant des résidus se sont déversés dans la rivière causant une pollution majeure du Gardon (DREAL, 2008). À $155 \mathrm{~cm}$ de profondeur, on peut observer une augmentation du facteur d'enrichissement du plomb (de 2,5 à 5, Figure 6). L'augmentation de la production minière de plomb entre 1870 et 1905 pourrait expliquer cette augmentation de métaux lourds entre les unités GE9 et GE11 (Figure 6). Par conséquent, en termes de chronologie relative, l'analyse géochimique indique que les unités GE1 à GE9, sont antérieures à 1870. Le FE du plomb est plus élevé dans les unités supérieures de la terrasse, autour de 9 dans GE18 et de 50 dans GE19. L'unité présentant un FE de 9 peut être liée à la forte augmentation de la production de plomb dans le milieu des années 1960 (GE18) et l'unité présentant un très grand FE (50) peut être associée à l'évènement de pollution majeur du bassin en 1976 (GE19, figure 6).

En plus des métaux traces, $d u{ }^{137} \mathrm{Cs}$ et $d u^{210} \mathrm{~Pb}_{\text {ex }}$ comme traceurs chronologiques, les crues extrêmes sont également très bien visibles sur les log litho-stratigraphiques. Ces traceurs chronologiques ont été comparés aux historiques des crues disponibles depuis 1890 à la station de jaugeage de Remoulins (données du SPC30). La comparaison de ces enregistrements permet d'attribuer de manière plus fine des âges aux dépôts de crues de la séquence GE (Figure 6). L'événement de 1958, le deuxième évènement le plus puissant enregistré par les archives instrumentales $\left(6400 \mathrm{~m}^{3} / \mathrm{s}\right)$, a déposé une unité de $25 \mathrm{~cm}$ d'épaisseur de sables moyens (GE16: $270 \mu \mathrm{m}$ ). Les trois unités sus-jacentes, GE17, GE18 et GE19 sont bien marquées par la pollution en plomb et par $1 \mathrm{e}^{137} \mathrm{Cs}$ et ont été affectées à trois évènements de plus faibles ampleurs (4000, 2900 et $3000 \mathrm{~m}^{3} / \mathrm{s}$ ) qui ont eu lieu en 1963,1969 et 1976 respectivement (Figure 6). Les couches sédimentaires associées à ces crues sont caractérisées par des sables plus fins. L'événement de 2002, le plus puissant jamais enregistré sur cette séquence, a déposé une unité de $30 \mathrm{~cm}$ d'épaisseur de sables moyens (GE20). Une corrélation semble exister entre l'intensité d'un évènement et la taille granulométrique qui caractérise son dépôt. L'enregistrement sédimentaire des crues avant 1958 semble incomplet, en effet on observe moins d'unités de dépôt de crue (7 unités depuis 1890) que d'évènement ayant une ampleur suffisante pour couvrir la terrasse (Figure 6). Sur la base des résultats du modèle hydraulique, environ 21 événements de crue auraient submergé la terrasse GE entre 1870 et 1958 dont 14 largement (Figure 5). En nous fondant sur une relation possible entre la granulométrie des sédiments et l'ampleur d'un évènement, GE15 pourrait être associé à 1951, GE14 à 1943, GE13 à 1933, GE12 à 1915, GE11 à 1907, GE10 à 1900 et GE9 à 1890 (Figure 6). Les processus d'érosion, des erreurs possibles dans les sources documentaires hydrologiques et les approximations dans le modèle pourraient également être à l'origine de cette plus faible corrélation entre enregistrement sédimentaire et enregistrement instrumental entre 1890 et 1958 . Pour conclure sur cette partie, il peut être souligné que la terrasse GE enregistre plutôt bien les évènements de crue du passé et qu'à priori, ce type d'objet géomorphologique peut être utilisé pour toute étude paléohydrologique sur le Gardon.

\section{IV.2.2. La cavité $G G$}

La cavité GG contient plus de 1,5 m de sédiments de dépôt de crue. Vingt-cinq unités sédimentaires ont été identifiées à l'oeil nu sur $0,90 \mathrm{~m}$ de remplissage sédimentaire, dont vingt-trois correspondant à des dépôts de crue (Figure 3 et 7). Ces dépôts se composent de sables à grains fins et moyens et des limons, présentant des laminations diffuses dans la partie supérieure et bien marquées dans la partie 


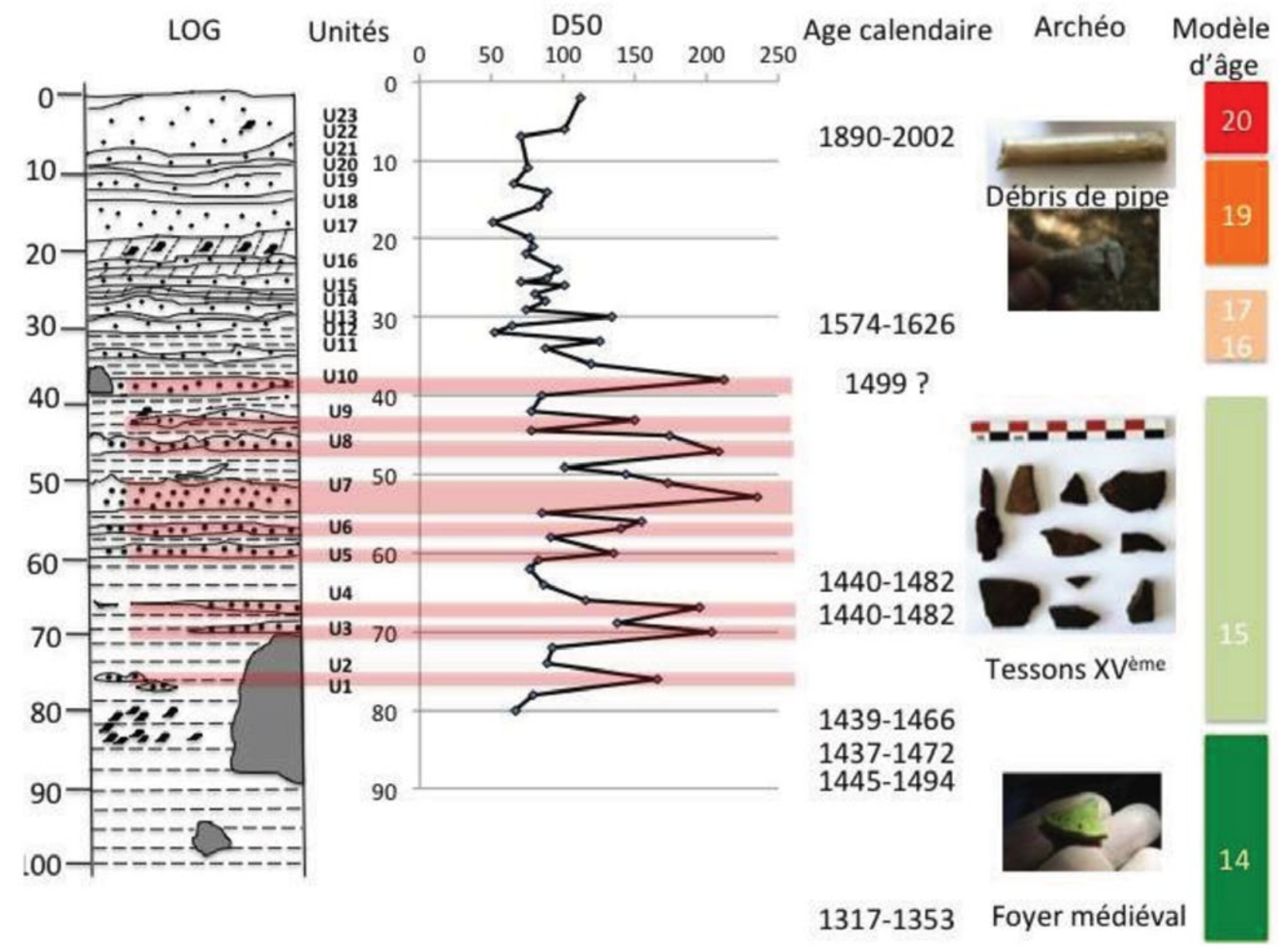

Figure 7 : Log de la coupe GG, unités de dépôts de crue, granulométrie (D50), âges 14C, objets archéologiques et modèle d'âge.

centrale de la coupe. La granulométrie moyenne (D50) est influencée par la présence d'apports anthropogènes liés à la fréquentation de la grotte par les hommes (charbons, foyers et épandages de cendre, fumier de grotte-bergerie) dans la partie supérieure de la coupe mais est contrôlée principalement par les dépôts de crue dans la partie inférieure et médiane de la coupe. Une analyse micromorphologique et géochimique fine (core scanner) est en cours pour détecter des signatures alluviales, pédogénétiques et anthropiques non visibles en stratigraphie et identifier l'origine des crues.

Le ${ }^{137} \mathrm{Cs}$ est présent dans les trois premiers centimètres (valeur de $7 \mu \mathrm{Bq} / \mathrm{g}$ ). Le même résultat est observé pour le ${ }^{210} \mathrm{~Pb}_{\text {ex }}$ (environ $20 \mu \mathrm{Bq} / \mathrm{g}$ ). Aucune activité en ${ }^{137} \mathrm{Cs}$ et ${ }^{210} \mathrm{~Pb}$ ex n'a été mesurée dans les dépôts plus anciens. La géochimie $\mathrm{du}$ profil indique que le facteur d'enrichissement (FE) du $\mathrm{Pb}$ varie dans une plage de 3 à 12. Les valeurs les plus basses (autour de 2,5) ont été mesurées sur la partie inférieure et médiane de la coupe. Le FE du Pb est plus élevé dans les unités supérieures à partir de $10 \mathrm{~cm}$. Le $\mathrm{XX}^{\mathrm{m} m e}$ siècle est donc représenté dans les 10 premiers $\mathrm{cm}$. Nous identifions probablement ici uniquement les 3 évènements majeurs du $20^{\text {ème }}$ siècle, c'est à dire 1907,1958 et 2002 . Le site GG étant localisé à une altitude plus importante $(15 \mathrm{~m})$ au dessus du lit de la rivière que le site GE $(10 \mathrm{~m})$, pour une morphologie similaire des Gorges dans cette zone, il est logique d'observer moins d'évènements. Ce résultat est cohérent avec ceux du modèle hydraulique pour lesquels il est démontré que seules les crues d'un débit supérieur à $4500 \mathrm{~m}^{3} / \mathrm{s}$ peuvent atteindre le site GG contre $2000 \mathrm{~m}^{3} / \mathrm{s}$ pour GE (Figure 5). Pour avoir une idée sur les âges des dépôts plus anciens 9 datations ${ }^{14} \mathrm{C}$ ont été réalisées sur charbons de bois à des profondeurs différentes (Figure 7). Les âges ${ }^{14} \mathrm{C}$ et la découverte sur le site de différents objets archéologiques permettent de mettre en place un modèle d'âge de la coupe GG (Figure 7).

Sur les 23 dépôts de crue identifiés, une dizaine d'événements peut être associée à la période $\mathrm{XV}^{\text {ème} / \text { début }}$ $\mathrm{XVI}^{\mathrm{ème}}$ siècle et une autre dizaine pour la période XVII/ $\mathrm{XX}^{\text {ème }}$ siècle (Figure 7). La chronologie et le nombre exact de lamines de crue reste encore à affiner mais 8-9 évènements peuvent déjà être associés au $X^{\text {ème }}$ siècle, 3-4 pour le XVI ${ }^{\text {ème }}, 4-5$ pour le XVII ${ }^{\text {ème }}$ et le XVIII ${ }^{\text {ème }}$, 4-5 pour

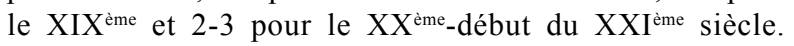
$\mathrm{D}$ 'après les résultats de la modélisation hydraulique, les nombreux évènements de crue recensés sur la période du $\mathrm{XV}^{\text {ème }}$ et $\mathrm{XVI}^{\mathrm{e} m e}$ siècle, correspondent à un débit supérieur à $4500 \mathrm{~m}^{3} / \mathrm{s}$ (valeur du débit permettant d'atteindre la cavité). D'autre part, si le lien entre la granulométrie (D50) et l'intensité des évènements est avéré alors il semble que les crues du XV-XVI ${ }^{\text {ème }}$ siècle aient été les plus intenses de ces 600 dernières années En tout cas, il semble que l'entrée dans le "Petit Age Glaciaire », induit une augmentation d'évènements intenses dans le Nord-Ouest du bassin méditerranéen. Ces résultats rejoignent les conclusions de Thorndycraft et al., (2005) sur des sites espagnols, qui suggéraient un impact large échelle des changements climatiques au $\mathrm{XV}^{\text {ème }}$ siècle sur l'Europe occidentale concernant les 
évènements de crue extrême. Cette conclusion est à confirmer par l'étude d'autres sites dans le Sud de la France.

\section{CONCLUSIONS}

Afin de reconstituer les crues anciennes du Gardon, nous avons étudié deux coupes sédimentaires. Ces coupes proviennent d'une terrasse alluviale (GE située à $10 \mathrm{~m}$ de hauteur au-dessus de la rivière) et d'une cavité karstique (GG à $15 \mathrm{~m}$ au-dessus de la rivière). Nous avons mis en évidence différents paléo-événements de crue qui ont été datés par des méthodes complémentaires $\left({ }^{137} \mathrm{Cs},{ }^{210} \mathrm{~Pb}_{\mathrm{ex}},{ }^{14} \mathrm{C}\right.$, métaux traces, objets archéologiques, historiques des crues). Nous montrons que la terrasse GE enregistre l'ensemble des évènements de crue entre 1958 et 2002 mais cet enregistrement semble incomplet avant 1958. En effet, on observe moins d'unités de dépôt de crue (7 unités depuis 1890) que d'évènements pluviométriques ayant une ampleur suffisante pour couvrir la terrasse. La position basse et peu protégée de cette séquence (à l'opposé de la cavité GG) explique sans doute un enregistrement incomplet, en raison des processus de troncatures lors des évènements les plus énergiques. Malgré cela, les terrasses sont des objets géomorphologiques à privilégier pour les études paléohydrologiques sur le Gardon. Nous montrons aussi que la coupe GG (cavité perche à $15 \mathrm{~m}$ au dessus de la rivière) offre un mode d'enregistrement des crues extrêmes et de datation particulièrement favorable, compte tenu de l'interstratification d'horizon d'occupation historiques homogènes, riches en charbons de bois peu à non remaniés, et de la protection par la paroi karstique face aux flux inondants qui favorise le processus d'aggradation. Cet enregistrement permet de reconstituer les évènements intenses (au dessus de $4500 \mathrm{~m}^{3} / \mathrm{s}$ ) au cours des 600 dernières années et illustre une augmentation des crues extrêmes dans notre région au cours du "Petit Age Glaciaire ». Elles sont plus particulièrement centrées sur le début de cette dernière phase de péjoration climatique, qui correspond à une période de transition et probablement de forte irrégularité climatique, entre une phase plus chaude (POM) et plus froide (PAG).

\section{REMERCIEMENTS}

Ce projet a été totalement financé par le projet ANR EXTRAFLO. Les auteurs souhaitent remercier Thierry Montecinos, Marie Bouchet, Stéphanie Garnero, Izabelle Avril, Cyril Soustelle pour leur aide sur le terrain, l'équipe technique de l'Irstea pour la réalisation des profils bathymétriques, la DDE de Nîmes pour les données historiques. Nous remercions le Laboratoire de Mesure ${ }^{14} \mathrm{C}$ (LMC14) ARTEMIS du CEA Saclay pour les analyses ${ }^{14} \mathrm{C}$.

\section{REFERENCES}

Benito G., Thorndycraft V.R. (2005) - Palaeoflood hydrology and its role in applied hydrological sciences. Journal of Hydrology. 313 :3-15

Bonnifait L., Delrieu G., Le Lay M., Boudevillain B., Masson A., Belleudy P., Gaume E., Saulnier G.-M. (2009) Hydrologic and hydraulic distributed modelling with radar rainfall input: Reconstruction of the 8-9 September 2002 catastrophic Flood event in the Gard region, France. Advances in Water Resources. 32 :1077-1089

Elbaz-Poulichet F., Bruneel O., Casiot C. (2006) - The Carnoules mine. Generation of As-rich acid mine drainage, natural attenuation processes and solutions for passive in-situ remediation. Documentation IRD. 1-8

El Kadi Abderrezzak, And K., Paquier A. (2009) One-dimensional numerical modeling of sediment transport and bed deformation in open channels. Water Resour. Res. 45 : W05404

Huet P.X., Martin J.L., Prime P., Foin C., Laurain P., Cannard (2003) - Retour d'expérience des crues de septembre 2002 dans les départements du Gard, de l'Hérault, du Vaucluse, des Bouches du Rhône, de l'Ardèche et de la Drôme. Rapport de l'Inspection Générale de l'Environnement. Ministre de l'Ecologie et du Développement Durable, République Française. Available at the Internet site: http://www.environnement.gouv. fr/infoprat/Publications/publi-ige.htm. $133 \mathrm{pp}$

Knox J.C., Daniels J.M. (2002) - Watershed scale and the stratigraphic record of large floods. House, P.K., Webb, R.H., Baker, V.R., and Levish, D. R., eds. Ancient Floods, Modern Hazards: Principles and Applications of Paleoflood Hydrology. Water Science and Application Series, American Geophysical Union. $5: 237-255$

Nuissier O., Ducroce V., Ricard D., Lebeaupin C., Anquetin S. (2008) - A Numerical Study of three catastrophic precipitating events over Southern France. I : Numerical Framework And Synoptic Ingredients. Quart. J. Roy. Meteor. Soc. 134 : $111-130$

Sheffer N. A., Y. Enzel G. Benito T. Grodek N. Poart M. Lang R. Naulet, $A N D$ D. Ceeur (2003) — Paleofloods and historical floods of the Ardèche River, France, doi:10.1029/2003WR002468, 12. Water Resour. Res. 39 :1376

Stuiver M, Reimer PJ. (1993) - Extended 14C data base and revised CALIB $3.014 \mathrm{C}$ age calibration program. Radiocarbon. 35(1) : 215-30

Thorndycraft V.R., Benito G., Rico M., Sopeña A., SÁnchez-Moya Y., Casas-Planes A. (2004) - A Late Holocene Paleoflood record from slackwater flood deposits of the Llobregat River, NE Spain. Journal Geological Society of India. 64 (4) :549-559

Thorndycraft V., Benito G., Rico M., Sopeña A., SÁnchez-Moya Y., CASAs A. (2005) - Paleoflood hydrology of the Llobregat River, NE Spain: a 3000 year record of extreme floods. Journal of Hydrology. 313(1-2) :16-31 